\title{
EDITORIAL
}

\section{Cobertura universal de salud en México: las brechas que persisten}

$\mathrm{C}$ on la reforma a la Constitución de los Estados Unidos Mexicanos en 1983, quedó formalizado en la máxima ordenanza legal del país el derecho a la protección de la salud. De esta forma inició un recorrido en términos de acceso a los servicios y a la protección financiera en salud que ha modificado de forma fundamental el panorama de la cobertura de protección en salud en México. Traducir la norma en aspectos prácticos ha sido un proceso mucho más complejo que el que permitió generar el consenso para colocar entre los derechos sociales el referente a la salud.

Durante el siglo XX México inició el desarrollo del sistema de salud con una lógica de crecimiento industrial, asociando la cobertura de protección en salud con esquemas de seguridad social por condición de empleo, esto es, servicios de salud con prepago provistos en un esquema público para los trabajadores en el sector formal de la economía. La realidad del desarrollo económico del país, distanciado de las proyecciones, generó un escenario con una elevada proporción de población en la economía informal, que al mismo tiempo se encontraba expuesta a choques económicos relacionados con gastos excesivos en salud que generaban empobrecimiento. En cifras recientes el empleo formal no se ha recuperado; si bien el desempleo ha reducido sus niveles máximos, sigue estando arriba de los niveles previos a la crisis de 2010, lo que ha incrementado la proporción de autoempleados o trabajadores sin prestaciones. ${ }^{1}$

La situación de la economía en general, con condiciones de crecimiento limitado y un importante trecho por recorrer para alcanzar la formalización general de la actividad económica, así como la evidencia del peso del gasto en salud, en particular en el segmento que se veía expuesto a gastos excesivos identificados como catastróficos, confirmaron el diagnóstico sobre el principal reto del sector salud mexicano: transitar hacia un esquema de separación de funciones, que a la vez implica integración dentro de las funciones. Es decir, ante un esquema con servicios de salud financiados con recursos públicos que operan de forma segmentada, moverse hacia una mayor eficiencia en la cobertura, manteniendo el máximo posible de competencia orientada a la calidad (elección de proveedor) en el sistema.

En ese contexto, con la reforma iniciada en 1995 con la descentralización de los servicios de salud dirigida a consolidar el papel rector en el nivel federal, y que con la creación del Sistema de Protección Social en Salud (SPSS) en 2003 se orienta hacia la protección financiera que al mismo tiempo genera acceso a los servicios de salud, se han establecido los mecanismos que deben permitir que todos los mexicanos cuenten con algún esquema de protección en salud. ${ }^{2}$

En "La búsqueda de la cobertura universal en salud: el logro de protección social para todos en México", uno de los artículos incluidos en este número de Salud Pública de México, Felicia Knaul encabeza a un destacado grupo de investigadores y funcionarios que informan sobre la cobertura en salud. A través de un análisis de diferentes elementos de información, los autores argumentan que para 2012 se ha alcanzado el objetivo de la cobertura de protección en salud en México, con cerca de $98 \%$ de todos los mexicanos afiliados a algún mecanismo de protección. Al mismo tiempo indican que si bien las cifras muestran que el gasto de bolsillo como porcentaje del gasto total en salud se ha reducido, éste sigue siendo cercano a la mitad del total, con 47 por ciento.

El artículo de Knaul y colaboradores, a la vez que hace un recorrido sobre lo que ha implicado este importante avance del sistema de salud en México, propone una ruta para los ajustes que siguen, es decir, para la continuación de la reforma que permita no sólo alcanzar la cobertura universal de protección en salud en términos de afiliación y acceso a un conjunto comprensivo de intervenciones, sino también a servicios efectivos, es decir, de calidad, que permitan traducir la utilización en ganancias en salud. 
En términos generales, la búsqueda a la que hace referencia el estudio se articula en el acceso a servicios efectivos (tanto en lo curativo como en lo preventivo) que no representen un empobrecimiento para las familias. Este ha sido el reto histórico: moverse de un esquema en el cual más de la mitad del gasto en salud se da de forma asociada con eventos de salud, mediante gasto de bolsillo y privado, hacia un esquema con acceso a servicios, ya sea de prepago o sin pago en el punto de contacto.

La ruta que ha seguido la reforma indica que se ha avanzado. Las cifras reportadas muestran una reducción importante en el porcentaje de hogares con gastos catastróficos en salud, que pasaron de $3.5 \%$ en 2000 a $2.7 \%$ en 2010, es decir, una reducción de $23 \%{ }^{3}$ No obstante, el que cerca de $50 \%$ del gasto total en salud sea todavía de ocurrencia en el evento como gasto de bolsillo y privado, indica que el reto sigue presente. Los datos de la Encuesta Nacional de Salud y Nutrición 2012 (ENSANUT 2012) sugieren que una parte de éste se traduce en la utilización de servicios privados ambulatorios e incluso hospitalarios por parte de población que cuenta con cobertura por algún tipo de seguro público, tanto para los que están en la seguridad social como en el SPSS. Analizar este aspecto llama a considerar aspectos relacionados con la calidad de los servicios públicos, tanto la técnica como la percibida. Es decir, el reto de la protección financiera va de la mano con el reto de la efectividad de los servicios. Los usuarios eligen servicios privados por la conveniencia en el acceso (posiblemente asociado con la calidad percibida) o por los resultados esperados, es decir, la expectativa de calidad técnica. ${ }^{4}$

Modificar el patrón de utilización puede repercutir en mayor eficiencia en el sistema, a la vez que favorecer la protección financiera de los hogares mexicanos. Las recomendaciones de la Organización para la Cooperación y el Desarrollo Económicos (OCDE) para México consideran esquemas orientados a reconocer el desempeño de los proveedores como mecanismo para incrementar la calidad de los servicios. ${ }^{1}$

Por otra parte, las cifras de cobertura de protección en salud que reporta la ENSANUT 2012 señalan que el porcentaje de población que no cuenta con protección se ha reducido de $60 \%$ reportado en 2000 a $21 \%$ en 2012. Estas cifras, diferentes a las que utilizan Knaul y colaboradores en el artículo mencionado, se explican por diversas razones. Una parte de la diferencia puede deberse a posibles errores de reporte por el hecho de que la ENSANUT 2012 obtiene la información para cada individuo a través de un único informante del hogar, que en algunos casos puede no contar con la información completa sobre la condición de aseguramiento de todos los integrantes. No obstante, esta diferencia llama a reforzar la integración de un padrón nominal de los servicios de salud en México, que de hecho es parte de la ruta a una mayor integración de los mismos.

A pesar de la posibilidad que implica el SPSS de asegurar la protección en salud de todos los mexicanos, la segmentación del sistema facilita los huecos en la incorporación al sistema de ciertos bloques de la población. Un esquema universal requiere de una mayor integración, que ciertamente no es un paso sencillo ante el peso de las instituciones existentes, con dinámicas y organizaciones no alineadas y con incentivos diversos.

Por lo que se refiere a la protección financiera, es necesario resaltar que como se ha mencionado aun si ha habido avances, de igual manera los hogares mexicanos siguen reportando un monto considerable de gasto en salud y aún hay hogares que reportan gastos catastróficos.

\section{Juan Pablo Gutiérrez*}

\section{Referencias}

I. OCDE. Perspectivas OCDE: México. Reformas para el cambio. Enero 2012. [consultado: 2012 sept 17]. Disponible en http://www.oecd.org/ mexico/49363879.pdf.

2. Knaul, F, Frenk J. Health Insurance In Mexico: Achieving Universal Coverage Through Structural Reform. Health Aff 2005;24(6): I467-I476. 3. Centro de Investigación Económica y Presupuestaria. Gasto Catastrófico por Motivos de Salud en México (ENIGH 2000-2010). México. CIEP, 2011.

4. Gutiérrez JP, Rivera-Dommarco J, Shamah-Levy T, Villalpando-Hernández S, Franco A, Cuevas-Nasu L, et al. Encuesta Nacional de Salud y Nutrición 2012. Resultados nacionales. Cuernavaca, México: Instituto Nacional de Salud Pública, 2012.

* Director del Centro de Investigación en Evaluación y Encuestas, Instituto Nacional de Salud Pública. Cuernavaca, Morelos, México 